\title{
Avaliação dos estressores para o paciente em uma unidade de terapia intensiva cardiológica
}

\section{Recebido em: 05/08/2013 \\ Aprovado em: 12/06/2014}

Fernanda Maria Nunes Mota Magalhães ${ }^{1}$

Sayonara Almeida de Jesus ${ }^{1}$

Cristiane Franca Lisboa Gois ${ }^{2}$

Eliana Ofelia LLapa-Rodríguez ${ }^{2}$

Valmira dos Santos ${ }^{3}$

Dayse Rosangela S Marques ${ }^{4}$

Maria Pontes de Aguiar Campos 5

Maria Cláudia Tavares de Mattos ${ }^{5}$

Gabryella Garibalde Santana Resende ${ }^{6}$

Resumo: O estudo objetivou identificar os fatores mais e menos estressores para os pacientes internados em uma Unidade de Terapia Intensiva (UTI). Trata-se de um estudo descritivo, realizado com 45 pacientes internados em uma UTI Cardiológica. Foi utilizada escala tipo Likert de 4 pontos para avaliar 42 possíveis estressores. Os fatores mais estressores são: não ter controle de si mesmo e cama e/ou travesseiros desconfortáveis, e os menos: ser examinado constantemente por médicos e enfermeiros e assistir aos cuidados médicos e de enfermagem realizados em outros pacientes. Conclui-se que os principais estressores estão relacionados a fatores intrapessoais e ambientais, assim como os menos estressores.

Descritores: estresse; unidades de terapia intensiva; cirurgia torácica; enfermagem.

\section{Evaluation of stressors to the patient in a cardiology intensive care unit}

Abstract: The study aimed to identify the most and the least stressful factors for patients hospitalized in a Intensive Care Unit (ICU). A descriptive study carried out with 45 patients hospitalized in a Cardiology ICU. A 4-point Likert scale was used to evaluate 42 possible stressors. The most stressful factors are: not to have control on oneself, uncomfortable bed and/or pillows, and the least stressful: being frequently examined by doctors and nurses, watching the medical and nursing care being carried out in other patients. We conclude that the main stressors are related to intrapersonal and environmental factors, and so are the least stressful ones.

Descriptors: stress; intensive care unit; thoracic surgery; nursing.

\section{Avaliación de estresores en pacientes de una unidad de cuidados intensivos cardiológicos}

Resumen: El estudio tuvo como objetivo identificar los factores más o menos estresantes, según la percepción del paciente internado en una Unidad de Cuidados Intensivos (UCl). Estudio descriptivo, desarrollado con 45 pacientes internados en una UCI Cardiológica. Se utilizó una escala tipo Likert de 4 puntos para evaluar 42 posibles causas de estrés. Los factores más estresantes fueron: no tener control de sí mismo, cama y/o almohada desconfortables, observar los cuidados médicos y de enfermería realizados en otros pacientes y escuchar el teléfono tocar. Concluimos que los principales estresantes presentes fueron los relacionados a factores intrapersonales y ambientales, así como considerados menos estresantes.

Descriptores: Lactancia materna, Validez de las pruebas, Enfermería en Salud Comunitaria.

\section{INTRODUÇÃO}

A Unidade de Terapia Intensiva se destina ao atendimento de pacientes que necessitam de cuidados intensivos, a qual guarda características próprias que a diferencia das demais unidades do hospital. Nesse setor coexistem fatores que têm sido considerados geradores de estresse para o paciente ${ }^{(1-3)}$.

Nesse sentido, Hans Selye (1956, p.34), pioneiro dos estudos sobre estresse no campo da biologia e da saúde humana, definiu ser o estresse,"o resultado não específico de qualquer demanda sobre o corpo, seja de efeito mental ou somático"(4). Selye observou que o estresse produzia reações de defesa e adaptação frente ao agente estressor e nominou a ocorrência do estresse de Síndrome Geral de Adaptação. Apesar de seus estudos terem sido motivados a partir de suas observações em pessoas doentes, Selye compreendeu que o estresse é parte normal do funcionamento do corpo, sendo uma conseqüência do ato de viver.

Dos estudos de Selye às pesquisas subseqüentes avolumam-se evidências da ação de outros agentes estressores de natureza não biológica e a influência das reações negativas a agentes estressores na saúde ${ }^{(5-7)}$. Sejam esses agentes estressores físico, mental, social, espiritual ou ambiental, podem desestabilizar a homeostase do indivíduo, com repercussão em outras dimensões, além da biológica ${ }^{(8)}$. Sob essa perspectiva de desestabilização a enfermeira Jean Watson ${ }^{(9)}$ corrobora com outros pesquisadores ao afirmar que $o$ estresse é um fator importante que causa desarmonia, afeta a saúde e desencadeia doença. Watson enfatiza que a compreensão das percepções que a pessoa tem da sua situação na qual se encontra ajuda a enfermeira a preparar um plano para aliviar o estresse do acontecimento.

A partir do exposto e da experiência profissional é possível afirmar que a hospitalização por si só já é um evento estressante e não raro considerada como experiência traumática. Acrescido a esse aspecto, tem-se a situação clínica do paciente e o comprometimento de suas necessidades básicas. O paciente durante a hospitalização

'Enfermeira graduada pela Universidade Tiradentes.

'Enfermeira. Doutora em Enfermagem. Professora Adjunta da Universidade Federal de Sergipe. Aracaju, Sergipe, Brasil.

${ }^{3}$ Enfermeira. Doutora. Professora da Faculdade de Sergipe - Fase, Aracaju, Sergipe, Brasil.

${ }^{4}$ Enfermeira. Professora da Universidade Tiradentes, Aracaju, Sergipe, Brasil.

${ }^{5}$ Enfermeira. Doutora. Professora Associada da Universidade Federal de Sergipe. Aracaju, Sergipe, Brasil.

${ }^{6}$ Enfermeira. Especialista em Educação profissional pela ENSP/FIOCRUZ. 
está fragilizado por se encontrar numa condição que lhe foi imposta de uma hora para outra, num ambiente novo e impessoal, onde seus costumes e horários pessoais nem sempre são seguidos, estão cercado por profissionais, pessoas diferentes, com as quais não convivem no seu dia a dia, gerando uma relação interpessoal muitas vezes "fria" e distante ${ }^{(10)}$. Como um plus a esse contexto que envolve a hospitalização, a possibilidade de vir a ser internado em uma UTI, um setor diferenciado por suas características físicas, equipamentos, rotina de assistência e estado de saúde dos pacientes assistidos, pode potencializar o estresse. A percepção do paciente com relação ao ambiente hospitalar influencia diretamente no seu estado de bem estar e segurança.

Na avaliação dos fatores estressantes para o paciente há de se considerar que a percepção do paciente nem sempre coincide com a dos profissionais que assistem aos pacientes nessas unidades, com os enfermeiros valorizando os estressores com maior intensidade do que os pacientes que vivenciam a permanência nessa unidade ${ }^{(11)}$

Dentre os três fatores que foram considerados como mais estressantes para os pacientes internados numa UTI cardiológica tem-se, em ordem decrescente"ter dor","não conseguir dormir","sentir falta do marido ou esposa"(2). Enquanto que para os profissionais de saúde os fatores para estressantes para o paciente são "estar amarrado por tubos", "ter tubos no nariz e/ou na boca", "não ter explicações sobre o tratamento"(1).

Considerando que a ciência da enfermagem focaliza o seu conhecimento no cuidado integral ao paciente, e que o estresse pode interferir na recuperação do paciente, pelo seu potencial de interferir na homeostase, o presente estudo teve por objetivo identificar os fatores mais e menos estressores, do ponto de vista de pacientes internados em uma UTI Cardiológica.

\section{MATERIAL E MÉTODO}

Trata-se de um estudo descritivo, exploratório, de abordagem quantitativa, realizado com pacientes internados em uma UTI Cardiológica de um hospital localizado no município de Aracaju, estado de Sergipe.

O projeto foi aprovado pelo Comitê de Ética em Pesquisa da Universidade Tiradentes mediante protocolo no 121010. A amostra foi, por conveniência, composta por 45 pacientes que atenderam aos critérios de inclusão: ter idade igual ou acima de 18 anos; ter permanecido internado na UTI no mínimo 48 horas; e estar consciente, alerta e orientado, e sem alteração neurológica ou psicológica no momento da entrevista.

O critério de permanência na UTI por pelo menos 48 horas, justifica-se por considerar necessário esse período para que o paciente esteja exposto ao ambiente e assimile a ação dos fatores potencialmente estressores presentes nessa unidade.

Para a coleta dos dados foram aplicados dois instrumentos, o primeiro para a coleta dos dados de identificação (idade, sexo e situação profissional) e clínicos (motivo da internação e tempo de internação). O segundo instrumento foi utilizado para a estratificação dos fatores estressores, a "Escala de Estressores em Unidade de Terapia Intensiva - EETI" (Intensive Care Unit Environmental Stressor Scale ICUESS) ${ }^{(12-13)}$, em sua versão traduzida e adaptada culturalmente para o português ${ }^{(14-15)}$. A EETI é composta por 42 questões que abordam vários fatores causadores de estresse para o paciente internado em UTI. Trata-se de escala do tipo Likert de quatro pontos, cada pergunta da escala possui quatro possibilidades de respostas com a seguinte pontuação: (1) significa não estressante, (2) pouco estressante, (3) estressante, (4) muito estressante. O intervalo possível para a escala é de 42 (42 questões $\times 1$ ) até $168(42 \times 4)$, sendo que, quanto maior o valor da somatória dos itens, maiores são os estressores possíveis.

A EETI tem sido considerada confiável para medir o conjunto dos 42 fatores potencialmente geradores de estresse. Entre os estudos que avaliaram a confiabilidade interna da escala, foi observado alfa de Cronbach entre 0,90 e 0,93, o que reflete a alta consistência interna dos itens da escala ${ }^{(1-2)}$.

Os dados foram processados e analisados em programa estatístico. Foram realizadas análises estatísticas descritivas de frequência simples para variáveis nominais/ categóricas, de tendência central (média) e de dispersão (desvio-padrão) para as variáveis contínuas. Um escore médio foi feito para cada um dos 42 itens da escala, classificados desde o mais até o menos estressor, a fim de obter o ranking dos fatores mais e menos estressores.

\section{RESULTADOS}

Foram entrevistados 45 pacientes que estavam internados em uma UTI Cardiológica. A média de idade dos pacientes foi de 49,20 anos. A maioria era do sexo masculino e não desempenhava atividade remunerada. $O$ motivo de internação mais freqüente foi para tratamento pós-operatório. Na amostra estudada obtivemos valores da EETI entre 43 e 100 (média de 65,17) (Tabela 1). A EETI demonstrou ser confiável, tendo apresentado um alfa de Cronbach igual a 0.88 .

Tabela 1- Caracterização da amostra. Aracaju, 2010.

\begin{tabular}{|c|c|c|c|c|c|}
\hline Variáveis & $\mathbf{N}$ & $\%$ & Intervalo & Média & D.P. \\
\hline Idade (em anos) & & & $22-79$ & 49,20 & 16,71 \\
\hline Sexo Masculino & 32 & 71,1 & & & \\
\hline \multicolumn{6}{|l|}{ Situação Profissional } \\
\hline Ativo & 20 & 44,5 & & & \\
\hline Aposentado & 14 & 31,1 & & & \\
\hline Outros & 11 & 24,4 & & & \\
\hline \multicolumn{6}{|l|}{ Motivo da internação } \\
\hline Pós-operatório & 35 & 77,8 & & & \\
\hline Tratamento clínico & 10 & 22,2 & & & \\
\hline $\begin{array}{l}\text { Tempo de internação } \\
\text { (em dias) }\end{array}$ & & & $2-40$ & 5,17 & 7,03 \\
\hline $\begin{array}{l}\text { Escala de estressores } \\
\text { (soma dos } 42 \text { itens) }\end{array}$ & & & $43-100$ & 65,17 & 16,08 \\
\hline
\end{tabular}

D.P. $=$ Desvio Padrão

Os fatores mais estressores para os sujeitos que fizeram parte da amostra foram, em ordem decrescente de grau de estresse:"não ter controle de si mesmo", "cama e/ou travesseiros desconfortáveis", "não conseguir dormir". Enquanto que os menos estressores foram: "ser examinado constantemente por médicos e enfermeiros", "assistir aos cuidados médicos e de enfermagem realizados em outros pacientes", "escutar o telefone tocar" (Tabela 2). 
Tabela 2 - Ranking dos estressores segundo amostra. Aracaju, 2010.

\begin{tabular}{|c|c|c|c|}
\hline 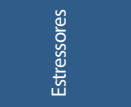 & 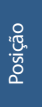 & $\begin{array}{l}\frac{00}{0} \\
\frac{0}{2}\end{array}$ & $\stackrel{\circ}{\circ}$ \\
\hline & $\bar{\sigma}$ & $\stackrel{\text { I }}{\text { N }}$ & $\stackrel{\stackrel{\leftrightarrow}{\sim}}{\sim}$ \\
\hline 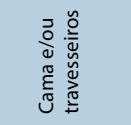 & $\tilde{\delta}$ & $\stackrel{m}{i}$ & $\stackrel{\tilde{m}}{\sim}$ \\
\hline 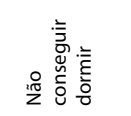 & m & $\underset{i}{\bar{i}}$ & 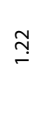 \\
\hline 홓 & d & $\underset{\text { i }}{\Delta}$ & 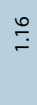 \\
\hline 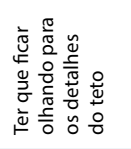 & $\ddot{n}$ & ọ & $\stackrel{m}{\rightleftarrows}$ \\
\hline 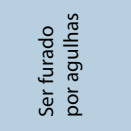 & ๑ & $\stackrel{\text { }}{-}$ & $\stackrel{\stackrel{\sim}{\longrightarrow}}{=}$ \\
\hline 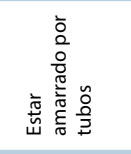 & $\hat{o}$ & $\stackrel{\infty}{\stackrel{\infty}{\sim}}$ & 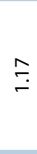 \\
\hline 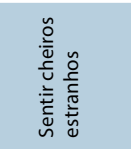 & 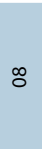 & $\underset{\sim}{\stackrel{\infty}{\Gamma}}$ & $\stackrel{\hat{o}}{r}$ \\
\hline 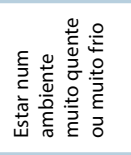 & o & $\stackrel{n}{\stackrel{\sim}{\sim}}$ & $\stackrel{n}{\rightleftarrows}$ \\
\hline 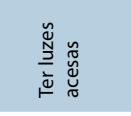 & $\circ$ & $\underset{+}{ז}$ & $\stackrel{\bar{\sigma}}{-}$ \\
\hline 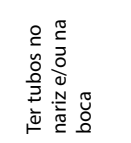 & $=$ & & $\stackrel{\circ}{r}$ \\
\hline 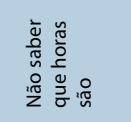 & $=$ & $\stackrel{\infty}{\stackrel{\infty}{\circ}}$ & 응 \\
\hline 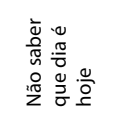 & $\simeq$ & م. & $\stackrel{\sim}{\sim}$ \\
\hline 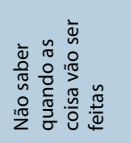 & $\simeq$ & مْ & $\stackrel{\hat{\sigma}}{-}$ \\
\hline 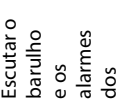 & $\underline{m}$ & مِّ & $\stackrel{\leftrightarrow}{r}$ \\
\hline
\end{tabular}

\begin{tabular}{|c|c|c|c|}
\hline 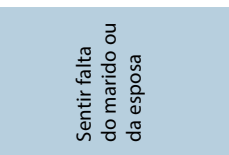 & $\underline{m}$ & $\stackrel{\widetilde{\sigma}}{-}$ & $\stackrel{\circ}{-}$ \\
\hline 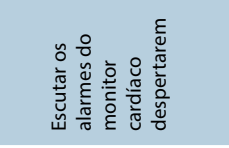 & \pm & 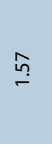 & $\stackrel{\bar{\sigma}}{\circ}$ \\
\hline 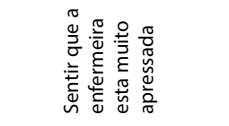 & \pm & $\stackrel{n}{n}$ & $\stackrel{\infty}{\circ}$ \\
\hline 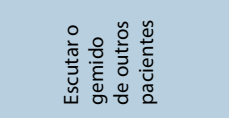 & 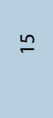 & $\stackrel{\leftrightarrow}{n}$ & ஃ̊․ \\
\hline 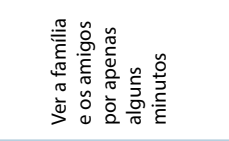 & 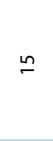 & $\stackrel{\leftrightarrow n}{\stackrel{n}{n}}$ & 范 \\
\hline 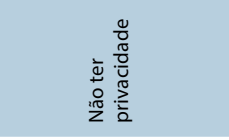 & $\stackrel{n}{\sim}$ & $\stackrel{\leftrightarrow}{\stackrel{n}{?}}$ & $\stackrel{\circ}{\infty}$ \\
\hline 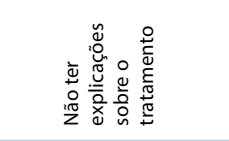 & $\stackrel{\circ}{\prime}$ & $\stackrel{5 n}{r}$ & $\stackrel{\sigma}{\Gamma}$ \\
\hline 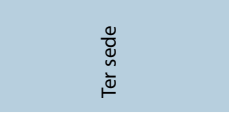 & $=$ & $\stackrel{\infty}{\leftrightarrows}$ & : \\
\hline 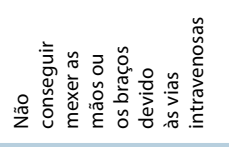 & $\stackrel{\infty}{\sim}$ & $\underset{\sim}{ \pm}$ & 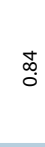 \\
\hline 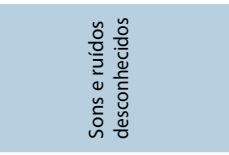 & $\stackrel{g}{ }$ & $\underset{f}{\stackrel{f}{-}}$ & $\stackrel{\infty}{\infty}$ \\
\hline 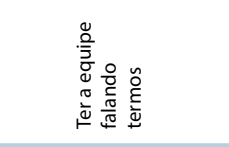 & 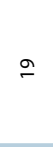 & $\stackrel{\Xi}{\stackrel{I}{*}}$ & $\stackrel{\infty}{\infty}$ \\
\hline 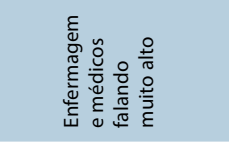 & i & $\stackrel{q}{g}$ & $\stackrel{\substack{\infty \\
\infty}}{\infty}$ \\
\hline 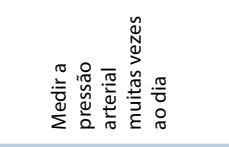 & $\bar{\sim}$ & $\stackrel{\hat{m}}{q}$ & ब. \\
\hline 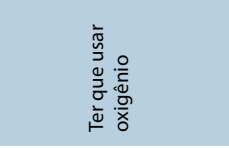 & $\bar{\sim}$ & $\hat{\underline{m}}$ & ז. \\
\hline 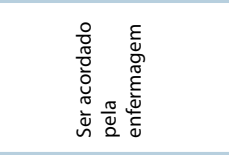 & $\bar{\sim}$ & $\stackrel{\hat{m}}{=}$ & ț \\
\hline 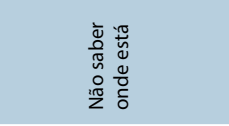 & $\approx$ & $\stackrel{m}{\stackrel{m}{-}}$ & 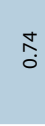 \\
\hline 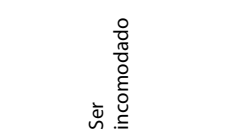 & $\approx$ & 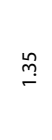 & : \\
\hline
\end{tabular}




\begin{tabular}{|c|c|c|c|}
\hline 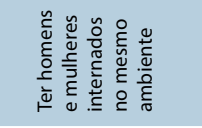 & $\tilde{\sim}$ & $\stackrel{\bar{m}}{\underline{m}}$ & $\stackrel{\circ}{\circ}$ \\
\hline 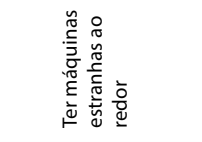 & $\stackrel{d}{d}$ & $\stackrel{\infty}{\stackrel{\infty}{~}}$ & $\begin{array}{l}\stackrel{n}{\hat{o}} \\
0\end{array}$ \\
\hline 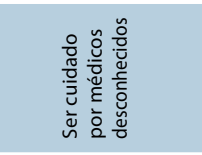 & $\stackrel{\sim}{\sim}$ & 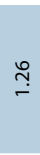 & స్. \\
\hline 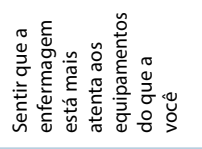 & $\stackrel{\circ}{\sim}$ & $\stackrel{\stackrel{t}{I}}{ }$ & $\stackrel{\circ}{\circ}$ \\
\hline 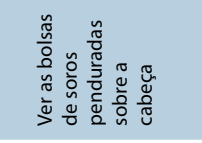 & $\hat{\sim}$ & $\stackrel{\simeq}{\simeq}$ & \\
\hline 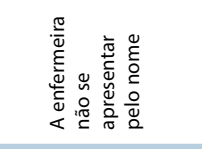 & $\stackrel{\infty}{\sim}$ & 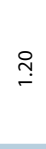 & $\stackrel{\widetilde{0}}{\circ}$ \\
\hline 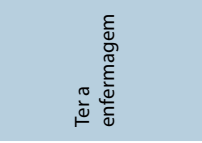 & $\stackrel{\infty}{\sim}$ & $\stackrel{\text { ㅁ }}{\longrightarrow}$ & 营 \\
\hline 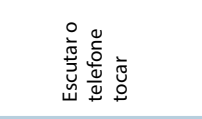 & ๙ి & 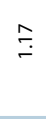 & in \\
\hline 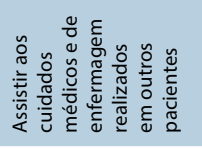 & $\stackrel{\circ}{m}$ & $\stackrel{n}{=}$ & fó. \\
\hline 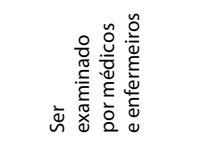 & $\bar{m}$ & $\stackrel{m}{=}$ & ֻั \\
\hline
\end{tabular}

\section{DISCUSSÃO}

Abordar o fenômeno estresse no contexto da assistência ao paciente é essencial para a promoção de um cuidado mais humanizado e com vistas a uma recuperação mais rápida e menos traumática. Nesse sentido, o estudo dos fatores estressores presentes numa UTI assume destaque pelas peculiaridades que envolvem a unidade, assistência desenvolvida e paciente assistido.

Assim, de acordo com os resultados da presente pesquisa, o fator não ter controle de si mesmo foi identificado como o principal estressor para os pacientes internados na UTI Cardiológica. Este resultado foi semelhante ao observado em outras pesquisas do gênero que identificou esse fator como um dos cinco mais estressores na opinião do paciente(2-3), tendo sido também relatado pelos familiares como um dos dez mais estressores(3). Uma das possíveis explicações para os resultados apresentados, talvez seja que no período de internação, os pacientes se considerem como altamente dependentes dos cuidados de outras pessoas.

O segundo fator considerado mais estressor foi cama e/ou travesseiro desconfortáveis, enquanto que em outros estudos este se posicionou após a $10^{a}$ colocação, tanto na opinião dos pacientes, quanto dos familiares e profissionais ${ }^{(1-3)}$. A mudança do ambiente quando o paciente é internado; o colchão coberto por tecido rígido, menos flexível às curvaturas do corpo; travesseiros com altura e textura diferente ao que costuma usar em sua casa, são fatores que podem interferir no sono, fato que pode justificar o fator não conseguir dormir ter sido considerado na amostra o terceiro mais estressante, resultado semelhante ao apresentado em outro estudo que identificou esse fator como o segundo no ranking na visão dos pacientes, e o quarto na dos familiares ${ }^{(15)}$ e equipe de saúde ${ }^{(1,15)}$. A privação do sono, assim como a sensação de dor, que foi considerado o quarto fator mais estressor, comprometem o paciente física e psicologicamente, amplificando com isso o estresse do paciente ${ }^{(3)}$. A dor foi identificada como um dos principais fatores estressores em outras pesquisas, tanto na visão do próprio paciente ${ }^{(2)}$, quanto dos familiares e profissionais que atuam em UT(1,3).

Os pacientes, sobretudo quando estão com a cânula endotraqueal, sentem ânsia de vômito o que causa dor devido ao esforço que fazem na musculatura torácica e abdominal ${ }^{(10)}$. Nesse sentido, um estudo realizado com pacientes, familiares e profissionais de saúde que atuavam em UTI para comparar a visão desses participantes quanto aos fatores estressores presentes em UTI, os autores identificaram que ter tubos no nariz e/ou na boca foi considerado o terceiro fator mais estressante na percepção dos pacientes e o segundo para os familiares e profissionais, e ter dor o primeiro, na percepção dos participantes dos três grupos ${ }^{(15)}$.

A dor representa um sintoma relevante para o paciente, no entanto, no dia a dia da assistência de enfermagem, muitas vezes ela é avaliada empiricamente, sem qualquer critério que a caracterize melhor e possibilite estratégias adequadas para eliminar ou diminuir os efeitos do fator etiológico ${ }^{(10)}$.

Diante do apresentado percebe-se que os maiores estressores na visão dos pacientes estão relacionados aos fatores intrapessoais e ambientais. Esses resultados podem funcionar como um guia para o planejamento da assistência com vistas à diminuição do estresse vivenciado pelos pacientes internados em UTI. Assim, considerando que os profissionais de enfermagem são os que permanecem por mais tempo em contato direto com o paciente, destaca-se a atuação desses profissionais no processo de busca pela humanização dessa unidade.

\section{CONCLUSÃO}

Para os pacientes que fizeram parte do estudo, os principais estressores presentes na UTI estão relacionados a fatores intrapessoais e ambientais:"não ter controle de si mesmo","cama e/ou travesseiros desconfortáveis", "não conseguir dormir", "ter dor". Do mesmo modo que os menos estressores: "Ser examinado por médicos e enfermeiros constantemente", "assistir aos cuidados médicos e de enfermagem realizados em outros pacientes", "escutar o telefone tocar" $\mathrm{e}$ "ter a enfermagem constantemente fazendo tarefas ao redor do leito".

A identificação dos fatores estressores é relevante para o planejamento da assistência aos pacientes, a fim de incluir estratégias que visem a diminuição do estresse por eles vivenciado durante a internação em UTI. 


\section{Referências}

1. Gois CFL, Dantas RAS - Estressores em uma unidade pósoperatória de cirurgia torácica: avaliação da enfermagem. Rev Latino-Am Enfermagem 2004; 12(1):22-7.

2. Marosti1 CA, Dantas RAS. Avaliação dos pacientes sobre os estressores em uma unidade coronariana. Acta Paul Enferm 2006; 19(2):190-5.

3. Bitencourt AGV, Neves FBCS, Dantas MP, Albuquerque LC, Melo RMV, Almeida AM, Agareno S, Teles JMM, Farias AMC, Messeder $\mathrm{OH}$. Análise de estressores para o paciente em Unidade de Terapia Intensiva. Rev Bras Ter Intensiva 2007; 19(1):53-9.

4. Selye H. The stress of life. New York: Mc Graw Hill, 1956 5. Cohen S, Tyrell DA, Smith AP. Psychological stress and susceptibility to the common cold. N Engl J Med 1991; 325(9):606-12.

6. Kiecolt-Glaser JK, Page GG, Marucha PT, MacCallum RC, Glaser R. Psychological influences on surgical recovery: perspectives from psychoneuroimmunology. Am Psychol 1998; 53(11): 120918

7. Bauer ME. Estresse: como ele abala as defesas do organismo. Ci Hoje 2002; 3(179):20-5.
8. Cross JR, Betty Neuman. In.: George et al. Teorias de Enfermagem os fundamentos da prática profissional. Tradução Maria Machado Garcez. Porto Alegre: Artes Médica, 1993.

9. Watson J. Conceptual for nursing pratice. Norwalk: Appeton Elange, 1998.

10. Gois CFL. O pós-operatório de cirurgia cardíaca na percepção de pacientes. [Mestrado em Promoção da Saúde] - Universidade de Franca, Franca; 2005. $75 \mathrm{f}$.

11. Cornock MA. Stress and the intensive care patient: perceptions of patients and nurses. J Adv Nurs 1998; 27(3):518-27.

12. Cochran J, Ganong LH. A comparison of nurses' and patients' perception of intensive care unit stressors. J Adv Nurs 1989; 14(12):1038-43.

13. Ballard KS. Identification of environmental stressors for patients in a surgical intensive care unit. Issues Ment Heath Nurs 1981; 1(3):89-108.

14. Novaes MA, Aronovich A, Ferraz MB, Knobel E. Stressors in ICU: patients' evaluation. Intensive Care Med. 1997; 23(12):1282-5.

15. Novaes MA, Knobel E, Bork AM. Estressores em UTI: Percepção do paciente, família e equipe de saúde. Intensive Care Med 1999; 25:1421-6. 